\title{
Safety and Efficacy of Durvalumab and Tremelimumab Alone or in Combination in Patients with Advanced Gastric and Gastroesophageal Junction Adenocarcinoma Ac
}

Ronan J. Kelly ${ }^{1,2}$, Jeeyun Lee ${ }^{3}$, Yung-Jue Bang ${ }^{4}$, Khaldoun Almhanna ${ }^{5}$, Mariela Blum-Murphy ${ }^{6}$, Daniel V.T. Catenacci ${ }^{7}$, Hyun Cheol Chung ${ }^{8}$, Zev A. Wainberg ${ }^{9}$, Michael K. Gibson ${ }^{10}$, Keun-Wook Lee ${ }^{11}$, Johanna C. Bendell ${ }^{12}$, Crystal S. Denlinger ${ }^{13}$, Cheng Ean Chee ${ }^{14}$, Takeshi Omori ${ }^{15}$, Rom Leidner ${ }^{16}$, Heinz-Josef Lenz $z^{17}$, Yee Chao ${ }^{18}$, Marlon C. Rebelatto ${ }^{19}$, Philip Z. Brohawn ${ }^{19}$, Peng He ${ }^{20}$, Jennifer McDevitt ${ }^{21}$, Siddharth Sheth ${ }^{21,22}$, Judson M. Englert ${ }^{21}$, and Geoffrey Y. Ku ${ }^{23}$

\section{ABSTRACT}

Purpose: This randomized, multicenter, open-label, phase Ib/II study assessed durvalumab and tremelimumab in combination or as monotherapy for chemotherapy-refractory gastric cancer or gastroesophageal junction (GEJ) cancer.

Patients and Methods: Second-line patients were randomized 2:2:1 to receive durvalumab plus tremelimumab (arm A), or durvalumab (arm B) or tremelimumab monotherapy ( $\operatorname{arm} \mathrm{C})$, and third-line patients received durvalumab plus tremelimumab (arm D). A tumor-based IFN $\gamma$ gene signature was prospectively evaluated as a potential predictive biomarker in second- and third-line patients receiving the combination (arm E). The coprimary endpoints were objective response rate and progression-free survival (PFS) rate at 6 months.

\section{Introduction}

Responses to approved treatments in the first- and second-line metastatic gastric cancer and gastroesophageal junction (GEJ) cancer settings are short lived, with virtually all patients experiencing disease

'The Sidney Kimmel Comprehensive Cancer Center, The Johns Hopkins University School of Medicine, Baltimore, Maryland. ${ }^{2}$ Charles A. Sammons Cancer Center, Baylor University Medical Center, Dallas, Texas. ${ }^{3}$ Division of Hematology-Oncology, Department of Medicine, Samsung Medical Center, Sungkyunkwan University School of Medicine, Seoul, Republic of Korea. ${ }^{4}$ Department of Internal Medicine, Seoul National University Hospital, Seoul National University College of Medicine, Seoul, Republic of Korea. ${ }^{5}$ Department of Medicine, Warren Alpert Medical School of Brown University, Providence, Rhode Island. ${ }^{6}$ Department of Gastrointestinal Medical Oncology, The University of Texas MD Anderson Cancer Center, Houston, Texas. ${ }^{7}$ Department of Medicine, Division of Hematology-Oncology, The University of Chicago Medicine Comprehensive Cancer Center, Chicago, Illinois. ${ }^{8}$ Division of Medical Oncology, Department of Internal Medicine, Yonsei Cancer Center, Yonsei University College of Medicine, Seoul, Republic of Korea. ${ }^{9}$ Division of HematologyOncology, Department of Medicine, Ronald Reagan UCLA Medical Center, Los Angeles, California. ${ }^{10}$ Division of Hematology and Oncology, Vanderbilt-Ingram Cancer Center, Vanderbilt University Medical Center, Nashville, Tennessee. ${ }^{11}$ Department of Internal Medicine, Seoul National University Bundang Hospital, Seoul National University College of Medicine, Seongnam, Republic of Korea.

${ }^{12}$ Sarah Cannon Research Institute/Tennessee Oncology, Nashville, Tennessee. ${ }^{13}$ Department of Hematology/Oncology, Fox Chase Cancer Center, Philadelphia, Pennsylvania. ${ }^{14}$ Department of Hematology-Oncology, National University Cancer Institute, Singapore. ${ }^{15}$ Department of Surgery, Osaka International Cancer Institute, Osaka, Japan. ${ }^{16}$ Earle A. Chiles Research Institute, Robert W. Franz Cancer Center, Providence Portland Medical Center, Providence Cancer
Results: A total of 113 patients were treated: 6 in phase $\mathrm{Ib}$ and 107 (arm A, 27; arm B, 24; arm C, 12; arm D, 25; arm E, 19) in phase II. Overall response rates were $7.4 \%, 0 \%, 8.3 \%, 4.0 \%$, and $15.8 \%$ in the five arms, respectively. PFS rates at 6 months were $6.1 \%, 0 \%, 20 \%, 15 \%$, and $0 \%$, and 12 -month overall survival rates were $37.0 \%, 4.6 \%, 22.9 \%, 38.8 \%$, and NA, respectively. Treatment-related grade $3 / 4$ adverse events were reported in $17 \%, 4 \%, 42 \%, 16 \%$, and $11 \%$ of patients, respectively.

Conclusions: Response rates were low regardless of monotherapy or combination strategies. No new safety signals were identified. Including use of a tumor-based IFN $\gamma$ signature and change in baseline and on-treatment circulating tumor DNA are clinically feasible and may be novel strategies to improve treatment response in this difficult-to-treat population.

progression $(1,2)$. For patients receiving two or more lines of treatment, options are limited, and treatment regimens involving novel approaches are urgently needed (3). Increasing understanding of tumor immunity and gastric cancer/GEJ cancer pathogenesis has

Institute, Portland, Oregon. ${ }^{17}$ Department of Preventive Medicine, Norris Comprehensive Cancer Center, University of Southern California, Los Angeles, California. ${ }^{18}$ Division of Medical Oncology, Center for Immuno-oncology, Department of Oncology, Taipei Veterans General Hospital, Taipei, Taiwan. ${ }^{19}$ Oncology Translational Medicine, AstraZeneca, Gaithersburg, Maryland. ${ }^{20}$ Early Oncology Statistics, AstraZeneca, Gaithersburg, Maryland. ${ }^{21}$ Early Oncology Clinical Development, AstraZeneca, Gaithersburg, Maryland. ${ }^{22}$ Division of Hematology and Oncology, University of North Carolina, Chapel Hill, North Carolina. ${ }^{23}$ Gastrointestinal Oncology Service, Department of Medicine, Memorial Sloan Kettering Cancer Center, New York, New York.

Note: Supplementary data for this article are available at Clinical Cancer Research Online (http://clincancerres.aacrjournals.org/).

Current address for J. McDevitt: Allogene Therapeutics, South San Francisco, California; and current address for J. Englert: UPMC Enterprises, Pittsburgh, Pennsylvania.

Corresponding Author: Ronan J. Kelly, Charles A. Sammons Cancer Center, Baylor University Medical Center, 3410 Worth Street, Suite 550, Dallas, TX 75246. Phone: 214-820-2828; Fax: 214-820-2846; E-mail: Ronan.Kelly@BSWHealth.org

Clin Cancer Res 2020;26:846-54

doi: 10.1158/1078-0432.CCR-19-2443

(C)2019 American Association for Cancer Research. 


\section{Translational Relevance}

In this randomized, multicenter phase $1 \mathrm{~b} / \mathrm{II}$ clinical study of metastatic/recurrent gastric or gastroesophageal junction cancer $(n=113)$, durvalumab plus tremelimumab demonstrated low response rates, a 12-month overall survival (OS) rate similar to that reported previously for checkpoint inhibitor combination, and a tolerable safety profile. Prospectively screening patients using a novel RNA-based IFN $\gamma$ signature from archival tissue was feasible, but this patient selection did not improve clinical response. Changes in circulating tumor DNA variant allele frequency correlated with median progression-free survival and OS, suggesting utility as an early indicator of clinical benefit.

fueled investigations of immune checkpoint inhibitors (ICI) in the setting of chemotherapy-refractory advanced or metastatic gastric cancer/GEJ cancer. Trials of anti-PD-1 and anti-CTL protein 4 (CTLA-4) ICIs (4-9), including pembrolizumab $(5,9)$ and nivolumab (7) as monotherapy and nivolumab in combination with ipilimumab, have shown durable clinical responses with acceptable toxicity profiles (6).

Although the antitumor effect and long-term durability observed in responders treated with ICI monotherapy and combination therapy are encouraging, the identification of novel predictive biomarkers to predict response is paramount. Approximately $40 \%$ of patients with gastric cancer/GEJ cancer express PD-L1 on tumor and immune cells $(5,10)$. In addition, microsatellite instability status (MSI) is associated with an improved prognosis as well as a reduced risk of lymph node metastasis, tumor invasion, and mortality $(11,12)$.

Interferon-gamma (IFN $\gamma$ ) produced by activated T cells and natural killer cells can directly upregulate PD-L1 expression and promote cytotoxicity through tumor-infiltrating macrophages recruitment, cytotoxic T-cell proliferation, and nitric oxide production. T-cell inflamed tumors show a high IFN $\gamma$ signature (13). An IFN $\gamma$ gene signature associated with improved response to pembrolizumab in multiple tumor indications, including gastric cancer/GEJ cancer has been identified (14). Similarly, patients with non-small-cell lung cancer receiving durvalumab with high expression of a four-gene signature comprising IFN $\gamma, C D 274, L A G 3$, and CXCL9 had higher overall response rates, longer progression-free survival (PFS), and improved overall survival (OS) than those with low expression, and these findings were replicated in an independent urothelial cancer cohort (15). Given the moderate-to-high expression of IFN $\gamma$ signature in a subset of gastric cancer/GEJ tumors, we performed a prospective evaluation of patients based on signature status.

The anti-PD-L1 antibody durvalumab has demonstrated durable clinical activity and a manageable safety profile in multiple tumor types, including gastric cancer/GEJ cancer (16-19). In addition, the anti-CTLA-4 antibody tremelimumab has the potential to interrupt a key coinhibitory signal, thus leading to T-cell activation in advanced gastric cancer/GEJ cancer $(10,20)$. In this study, we investigated the potential clinical benefits of durvalumab and tremelimumab in combination and as monotherapy in chemotherapy-refractory advanced gastric cancer/GEJ cancer. We also prospectively evaluated the ability of a tumor-based IFN $\gamma$ gene signature to identify a subset of secondand third-line patients with gastric cancer/GEJ cancer who were most likely to respond to dual ICI therapy.

\section{Patients and Methods}

\section{Study design and treatments}

This study is registered with ClinicalTrials.gov (NCT02340975) and was conducted at 30 centers globally, including sites in Canada (2), Japan (3), Korea (4), Singapore (2), Taiwan (3), and the United States (16), from March 2015 to January 2018. The study protocol and amendments were approved by local institutional boards and performed in accordance with the Declaration of Helsinki and is consistent with International Conference on Harmonisation, Good Clinical Practice, and applicable regulatory requirements. All patients provided written informed consent.

The phase Ib portion of the study $(N=6)$ involved a safety run-in assessment of durvalumab and tremelimumab at doses and schedules selected for dose expansion in patients with gastric or GEJ adenocarcinoma. Patients received durvalumab $20 \mathrm{mg} / \mathrm{kg}$ plus tremelimumab $1 \mathrm{mg} / \mathrm{kg}$ i.v. every 4 weeks (Q4W) for four cycles, followed by durvalumab $10 \mathrm{mg} / \mathrm{kg}$ every 2 weeks (Q2W) for up to 12 months (16). Patients were monitored for dose-limiting toxicities before enrollment in phase II (Supplementary Fig. S1).

In the phase II portion $(N=88), 63$ patients with disease refractory to one platinum- or fluoropyrimidine-based chemotherapy regimen were randomized 2:2:1 to one of three second-line treatment arms: A, B, or C. Third-line patients who had progressed on two regimens enrolled in arm $\mathrm{D}(n=25)$. Patients in all arms were treated for up to 12 months. Patients in arms A and D received durvalumab $20 \mathrm{mg} / \mathrm{kg}$ plus tremelimumab $1 \mathrm{mg} / \mathrm{kg}$ Q4W for four cycles, followed by durvalumab $10 \mathrm{mg} / \mathrm{kg}$ Q2W. Patients in arm B received durvalumab monotherapy $(10 \mathrm{mg} / \mathrm{kg}) \mathrm{Q} 2 \mathrm{~W}$. Patients in arm C received tremelimumab monotherapy $(10 \mathrm{mg} / \mathrm{kg}) \mathrm{Q} 4 \mathrm{~W}$ for seven doses and then every 12 weeks for two doses (for a total of up to 9 doses). Patients in the durvalumab and tremelimumab monotherapy arms could cross over to combination therapy at the time of disease progression if they met specific criteria. Those who did not have progression during the first 12 months and entered follow-up were eligible for retreatment for up to 12 months at the time of progression.

An additional arm (arm E; $n=19$ ) was added per a study amendment to prospectively evaluate the ability of a tumor-based IFN $\gamma$ gene signature in second- and third-line patients to identify patients with an increased likelihood of response. Prescreening was undertaken using archival formalin-fixed paraffin-embedded tissue to assign IFN $\gamma$ signature status with a custom, targeted RNA sequencing assay using Ion AmpliSeq (Thermo Fisher Scientific) sequencing technologies. The positive cutoff was established at the upper tertile of IFN $\gamma$ expression from an existing dataset, including data from arms A-D (15). Patients who were positive were eligible to be screened upon progression while receiving first- or second-line therapy. Patients enrolled in this arm received durvalumab $20 \mathrm{mg} / \mathrm{kg}$ and tremelimu$\mathrm{mab} 1 \mathrm{mg} / \mathrm{kg}$ i.v. Q4W for four cycles, followed by durvalumab $10 \mathrm{mg} / \mathrm{kg}$ Q2W for $\leq 12$ months.

A prespecified interim analysis was performed on arms A and B after 20 patients were enrolled and followed for $\geq 8$ weeks to assess whether the criterion for expansion $[\geq 2$ of 20 patients with complete response (CR), partial response (PR), or stable disease (SD) for 8 weeks] was met. If the criterion was not met, enrollment in arms A, B, and C could be stopped. Preliminary biomarker data could be used to support the decision to continue or stop enrollment. Similar interim analyses were planned for arm D (enrollment could stop if $\leq 2$ of 25 patients had CR or PR) and arm E (enrollment could stop if $\leq 3$ of 20 patients had $\mathrm{CR}$ or PR). 


\section{Patients}

Key inclusion criteria included histologic or cytologic confirmation of metastatic or recurrent gastric or GEJ adenocarcinoma that progressed after $\leq 2$ lines of systemic platinum- or fluoropyrimidine-based chemotherapy and presence of a measurable lesion amenable to biopsy. Key exclusion criteria included HER2-overexpressing metastatic or recurrent gastric cancer/GEJ cancer; active or prior documented autoimmune or inflammatory disease within 3 years; and prior treatment with any immunotherapy.

\section{Study assessments}

The coprimary efficacy endpoints for the phase II portion were the confirmed objective response rate (ORR) and PFS at 6 months (PFS-6) based on investigator assessment according to Response Evaluation Criteria in Solid Tumors (RECIST) version 1.1. Clinical activity was assessed by imaging every 8 weeks through end of treatment.

Secondary endpoints included the safety of durvalumab and tremelimumab in combination, durvalumab monotherapy, and tremelimumab monotherapy, including adverse events (AE) and serious AEs based on laboratory parameters, vital signs, and electrocardiograms. AEs were assessed and graded using the National Cancer Institute Common Terminology Criteria for AEs, version 4.0 (21). Clinical activity of durvalumab and tremelimumab in combination and as monotherapy was further assessed by disease control rate, duration of response, PFS, and OS.

PD-L1 expression was determined by IHC [Ventana PD-L1 (SP263) assay] and analyzed for correlation with clinical activity of durvalumab and tremelimumab. PD-L1 expression was determined to be positive if the proportion of tumor cells with staining at any intensity was $\geq 1 \%$.

\section{Prognostic scores}

Prognostic scores were retrospectively calculated to characterize the baseline features of the different treatment arms in phase II and allow retrospective analysis. These scores included the Royal Marsden Hospital (RMH) prognostic score (22) and the Gustave-Roussy Immune (GRIm)-score (23). The RMH score is based on three objective variables: number of metastatic sites ( $<3$ or $\geq 3$ sites), lactate dehydrogenase level [less than the upper limit of normal (ULN) or greater than or equal to the ULN], and serum albumin level $(<3.5$ or $\geq 3.5 \mathrm{~g} / \mathrm{dL}$ ). For each variable, the score was validated in patients treated with cytotoxic and targeted therapies (22). Patients with a lower RMH score at baseline ( 0 or 1 ) had a longer median OS than patients with a poor prognostic score (2 or 3 ). The GRIm score is based on serum albumin level, lactate dehydrogenase level, and neutrophil-tolymphocyte ratio. Patients with a high GRIm score $(>1)$ had inferior OS compared with those with a lower score $(0$ or 1$)$. This has been prospectively validated for patients in phase 1 trials receiving immunotherapy (23).

\section{Circulating tumor DNA}

A 73-gene panel measured DNA variants using the Guardant360 assay in plasma circulating tumor DNA (ctDNA). Treatment arms were combined to demonstrate associations of changes in mutation variant allele frequencies (VAF) with clinical response. Mutation VAFs and total mutation count of patient single-nucleotide variants and insertions/deletions were correlated with clinical outcomes. ORR was calculated according to RECIST 1.1. Cox proportional HRs were calculated while adjusting for age, sex, Eastern Cooperative Oncology Group performance status, line of therapy, and treatment arm.

\section{Statistical analyses}

All analyses were performed for the as-treated population, which included patients who received one or more dose of any study drug. The coprimary efficacy endpoints of ORR and PFS- 6 were based on RECIST 1.1. For ORR, the two-sided 95\% exact confidence intervals (CI) were estimated using binomial distribution. For PFS-6, KaplanMeier estimates were provided along with two-sided 95\% CIs. Sample size determination was based primarily on providing sufficient precision for estimating ORR and PFS-6. The targeted ORR range was approximately $35 \%$; with full enrollment the study would have had $85 \%$ power to detect a difference in ORR of $35 \%$ in arm A and $10 \%$ in arm B. The targeted PFS- 6 rate was $53 \%$, corresponding to a median PFS of 6.6 months or a $50 \%$ improvement over the median PFS reported for ramucirumab + paclitaxel (24).

\section{Results}

At the data cutoff of August 24, 2018, 107 patients had been enrolled in the phase II portion. Before the phase II portion, six patients were enrolled in a phase $\mathrm{Ib}$ safety run-in period. Three patients received one prior line of therapy, and the remaining had two or more lines. Although no responses were observed, two patients had SD (Supplementary Table S1). Five of six patients had a treatment-related AE (TRAE).

Fifty-two patients had received durvalumab in combination with tremelimumab (27 second line and 25 third line), 24 had received durvalumab monotherapy, and 12 had received tremelimumab monotherapy. The median duration of follow-up was 9.2, 3.5, 9.2, and 10.7 months in arms A, B, C, and D, respectively. An additional 19 second- or later-line patients received durvalumab and tremelimumab in the IFN $\gamma$-high group. Baseline characteristics of patients in arms A through E are shown in Table 1.

At the prespecified interim analysis, further enrollment in arms A, $\mathrm{B}$, and $\mathrm{C}$ was stopped at the sponsor's discretion despite meeting the prespecified criteria for expansion, based on the evolving immunotherapy landscape in gastric cancer/GEJ cancer. The discontinuation of enrollment in arms A, B, and C was not due to safety concerns. Similarly, an interim analysis on data for 19 patients in arm E also led to discontinuation of enrollment for that cohort.

\section{Efficacy}

No significant differences among the treatment arms were observed in ORR and PFS (Table 2; Supplementary Fig. S2). The confirmed ORR was $7.4 \%$ (two of 27 patients; $95 \%$ CI, $0.9 \%-24.3 \%$ ) in arm A compared with $0 \%$ in arm B (durvalumab monotherapy; 0 of 24 patients; $95 \% \mathrm{CI}, 0 \%-14.2 \%$ ) and $8.3 \%$ in arm C (tremelimumab monotherapy; one of 12 patients; $95 \%$ CI, $0.2 \%-38.5 \%$; Table 2; Fig. 1). The PFS rate at 6 months was $6.1 \%$ (95\% CI, $0.5 \%-22.2 \%)$, $0 \%$ (95\% CI, NA), and $20.0 \%$ (95\% CI, 3.1\%-47.5\%), respectively.

The median OS was 9.2 months (95\% CI, 5.4-12.6 months), 3.4 months (95\% CI, 1.7-4.4 months), and 7.7 months (95\% CI, 2.1-13.7 months) in the combination therapy (arm A), durvalumab monotherapy (arm B), and tremelimumab monotherapy (arm C) arms, respectively (Fig. 2). The OS rate at 12 months in the combination therapy arm (arm A) was 37.0\% (95\% CI, 19.6\%-54.6\%) compared with $4.6 \%$ (95\% CI, $0.3 \%-19.0 \%$ ) and $22.9 \%$ (95\% CI, $3.5 \%-52.4 \%)$ in the durvalumab monotherapy and tremelimumab monotherapy arms, respectively. The OS rate at 24 months was $18.5 \%$ (95\% CI, 6.7\%-34.8\%) in the combination therapy arm, $0 \%$ in the durvalumab monotherapy arm, and $11.5 \%$ (95\% CI, $0.6 \%-39.6 \%)$ in the tremelimumab monotherapy arm. 
Table 1. Baseline characteristics.

\begin{tabular}{|c|c|c|c|c|c|}
\hline $\begin{array}{l}\text { Characteristics } \\
\text { No. of patients }\end{array}$ & $\begin{array}{l}\text { Arm A } \\
2 L D+T \\
(n=27)\end{array}$ & $\begin{array}{l}\text { Arm B } \\
2 L D \\
(n=24)\end{array}$ & $\begin{array}{l}\text { Arm C } \\
2 \mathrm{~L} \mathrm{~T} \\
(n=12)\end{array}$ & $\begin{array}{l}\text { Arm D } \\
3 L D+T \\
(n=25)\end{array}$ & $\begin{array}{l}\text { Arm E } \\
2 \mathrm{~L} / 3 \mathrm{~L} D+\mathrm{T} \text { IFN } \gamma+ \\
(n=19)\end{array}$ \\
\hline Median age (range), years & $64.0(27-78)$ & $60.0(29-79)$ & $54.0(19-74)$ & $59.0(35-75)$ & $57.0(32-82)$ \\
\hline Male, $n(\%)$ & $20(74.1)$ & $19(79.2)$ & $8(66.7)$ & $16(64.0)$ & $14(73.7)$ \\
\hline \multicolumn{6}{|l|}{ Race, $n(\%)^{a}$} \\
\hline Asian & $7(25.9)$ & $11(45.8)$ & $5(41.7)$ & $12(48.0)$ & $13(68.4)$ \\
\hline Other & 0 & 0 & $1(8.3)$ & 0 & 0 \\
\hline White & $20(74.1)$ & $13(54.2)$ & $6(50.0)$ & $13(52.0)$ & $6(31.6)$ \\
\hline \multicolumn{6}{|l|}{ Extent of disease at study entry, $n(\%)^{\mathrm{b}}$} \\
\hline Locoregionally recurrent only & $2(7.4)$ & $1(4.2)$ & $2(16.7)$ & 0 & 0 \\
\hline Locoregionally recurrent with metastasis & $14(51.9)$ & $14(58.3)$ & $2(16.7)$ & $13(52.0)$ & $9(47.4)$ \\
\hline Metastasis without locoregional recurrence & $11(40.7)$ & $9(37.5)$ & $8(66.7)$ & $12(48.0)$ & $10(52.6)$ \\
\hline \multicolumn{6}{|l|}{ PD-L1 status, $n(\%)^{\mathrm{c}}$} \\
\hline $\mathrm{High}^{\mathrm{d}}$ & $14(51.9)$ & $9(37.5)$ & $6(50.0)$ & $5(20.0)$ & $9(47.4)$ \\
\hline Low/negative & $10(37.0)$ & $11(45.8)$ & $4(33.3)$ & $15(60.0)$ & $3(15.8)$ \\
\hline Unknown ${ }^{\mathrm{e}}$ & $3(11.1)$ & $4(16.7)$ & $2(16.7)$ & $5(20.0)$ & $7(36.8)$ \\
\hline \multicolumn{6}{|l|}{ RMH score, $n(\%)^{\mathrm{f}}$} \\
\hline 0 & $15(55.6)$ & $5(20.8)$ & $6(50.0)$ & $11(44.0)$ & $5(26.3)$ \\
\hline 1 & $6(22.2)$ & $4(16.7)$ & $3(25.0)$ & $9(36.0)$ & $7(36.8)$ \\
\hline 2 & $6(22.2)$ & $15(62.5)$ & $3(25.0)$ & $4(16.0)$ & $5(26.3)$ \\
\hline 3 & 0 & 0 & 0 & 0 & $2(10.5)$ \\
\hline Unknown & 0 & 0 & 0 & $1(4.0)$ & 0 \\
\hline \multicolumn{6}{|l|}{ GRIm score, $n(\%)^{f}$} \\
\hline 0 & $12(44.4)$ & $7(29.2)$ & $3(25.0)$ & $15(60.0)$ & $7(36.8)$ \\
\hline 1 & $12(44.4)$ & $10(41.7)$ & $8(66.7)$ & $6(24.0)$ & $6(31.6)$ \\
\hline 2 & $1(3.7)$ & $4(16.7)$ & $1(8.3)$ & $2(8.0)$ & $5(26.3)$ \\
\hline 3 & 0 & $2(8.3)$ & 0 & $1(4.0)$ & $1(5.3)$ \\
\hline Unknown & $2(7.4)$ & $1(4.2)$ & 0 & $1(4.0)$ & 0 \\
\hline
\end{tabular}

${ }^{\mathrm{a} E a c h}$ race category counts patients who selected only that category. bInformation is missing for one patient in arm B.

${ }^{\mathrm{C} P D-L 1}$ expression was determined using the VENTANA SP263 assay.

${ }^{d}$ PD-L1 high was defined as $\geq 1 \%$ tumor cells at baseline.

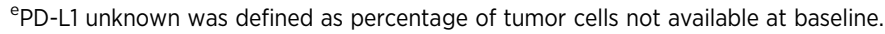

${ }^{f} \mathrm{RMH}$ or GRIm score of 2 or 3 was classified as a high score.

Table 2. Efficacy endpoints.

\begin{tabular}{|c|c|c|c|c|c|}
\hline Endpoint & $\begin{array}{l}\text { Arm A } \\
2 L D+T \\
(n=27)\end{array}$ & $\begin{array}{l}\text { Arm B } \\
2 \mathrm{~L} \mathrm{D} \\
(n=24)\end{array}$ & $\begin{array}{l}\text { Arm C } \\
2 \mathrm{~L} \mathrm{~T} \\
(n=12)\end{array}$ & $\begin{array}{l}\text { Arm D } \\
3 L D+T \\
(n=25)\end{array}$ & $\begin{array}{l}\text { Arm E } \\
2 \mathrm{~L} / 3 \mathrm{~L} D+\mathrm{T} \text { IFN } \gamma+ \\
(n=19)\end{array}$ \\
\hline ORR, $n(\% ; 95 \% \mathrm{Cl})$ & $2(7.4 ; 0.9-24.3)$ & $0(0 ; 0-14.2)$ & $1(8.3 ; 0.2-38.5)$ & 1 (4.0; 0.1-20.4) & $3(15.8 ; 3.4-39.6)$ \\
\hline Complete response & 0 & 0 & 0 & 0 & $1(5.3)$ \\
\hline Partial response & $2(7.4)$ & 0 & $1(8.3)$ & $1(4.0)$ & $2(10.5)$ \\
\hline Stable disease & $5(18.5)$ & $3(12.5)$ & $2(16.7)$ & $6(24.0)$ & $1(5.3)$ \\
\hline Progressive disease & $15(55.6)$ & $14(58.3)$ & $5(41.7)$ & $14(56.0)$ & $12(63.2)$ \\
\hline Nonevaluable ${ }^{a}$ & $5(18.5)$ & $7(29.2)$ & $4(33.3)$ & $4(16.0)$ & $3(15.8)$ \\
\hline Ongoing response rate & $2(100)$ & NA & 0 & 0 & $1(33.3)$ \\
\hline $\mathrm{DCR}, n(\% ; 95 \% \mathrm{Cl})$ & $7(25.9 ; 11.1-46.3)$ & $3(12.5 ; 2.7-32.4)$ & $3(25.0 ; 5.5-57.2)$ & 7 (28.0; 12.1-49.4) & $4(21.1 ; 6.1-45.6)$ \\
\hline DCR24, $n(\% ; 95 \% \mathrm{Cl})$ & $2(7.4 ; 0.9-24.3)$ & $0(0 ; 0-14.2)$ & 3 (25.0; 5.5-57.2) & $3(12.0 ; 2.5-31.2)$ & $3(15.8 ; 3.4-39.6)$ \\
\hline Median time to response, weeks (95\% Cl) & $16.1(8.1-24.0)$ & NA & 11.6 (NA-NA) & 31.1 (NA-NA) & $7.7(7.3-8.0)$ \\
\hline Median duration of response $(95 \% \mathrm{Cl})$, weeks & NA & NA & 20.1 (NA-NA) & 32.3 (NA-NA) & $13.3(8.1-N A)$ \\
\hline Median PFS (95\% Cl), months & $1.8(1.6-3.3)$ & $1.6(1.0-1.8)$ & $1.7(0.8-5.3)$ & $1.8(1.6-3.5)$ & $1.8(1.6-1.9)$ \\
\hline PFS-6 (95\% Cl), \% & $6.1(0.5-22.2)$ & NA & $20.0(3.1-47.5)$ & $15.0(4.0-32.6)$ & NA \\
\hline Median OS (95\% Cl), months & $9.2(5.4-12.6)$ & $3.4(1.7-4.4)$ & $7.7(2.1-13.7)$ & $10.6(4.8-14.8)$ & $7.0(2.4-7.5)$ \\
\hline OS-12 (95\% Cl), \% & $37.0(19.6-54.6)$ & $4.6(0.3-19.0)$ & $22.9(3.5-52.4)$ & $38.8(19.2-58.1)$ & NA \\
\hline OS-24 (95\% Cl), \% & $18.5(6.7-34.8)$ & $0(0-0)$ & $11.5(0.6-39.6)$ & $9.7(1.7-26.5)$ & NA \\
\hline
\end{tabular}

Abbreviations: 2L, received treatment in the second-line setting; $3 \mathrm{~L}$, received treatment in the third-line setting; $D$, durvalumab; DCR, disease control rate (complete response + partial response + stable disease); DCR24, DCR at 24 weeks (complete response + partial response + stable disease $\geq 24$ weeks); NA, not applicable; OS-12, OS rate at 12 months; OS-24, OS rate at 24 months; T, tremelimumab.

${ }^{a}$ Nonevaluable includes patients with only a baseline assessment or a response of complete response, partial response, or stable disease at an interval of $<54$ days (the minimum criteria for stable disease duration) without subsequent disease evaluation and patients who had all overall response evaluations assessed as nonevaluable. 
A

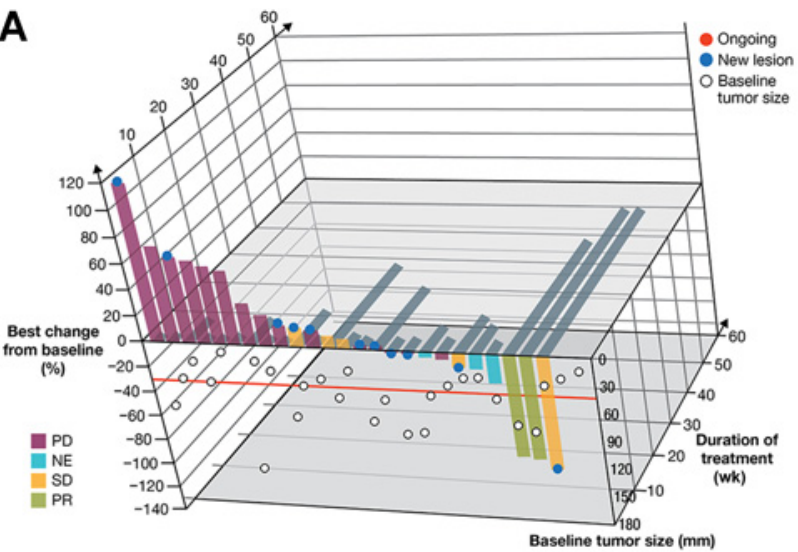

C

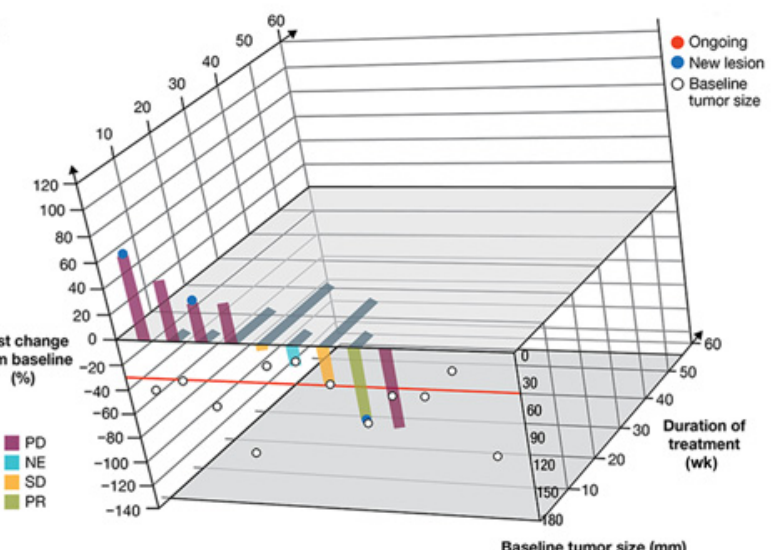

E

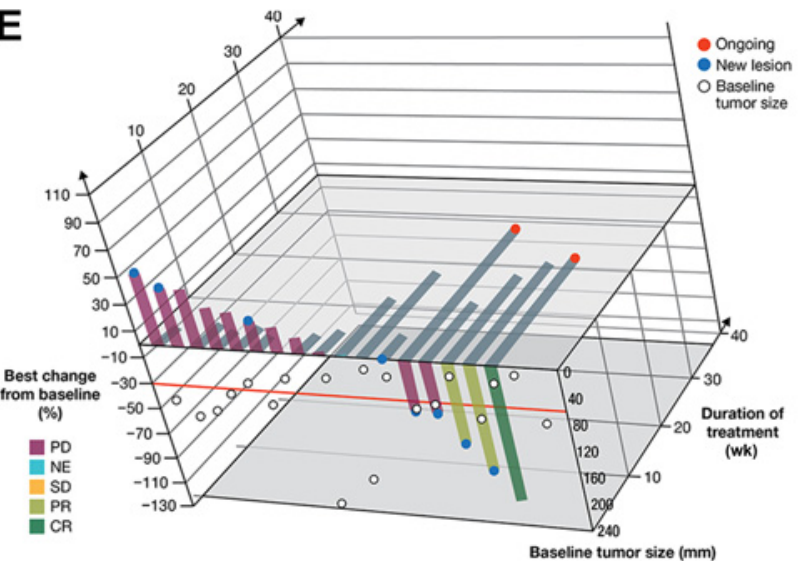

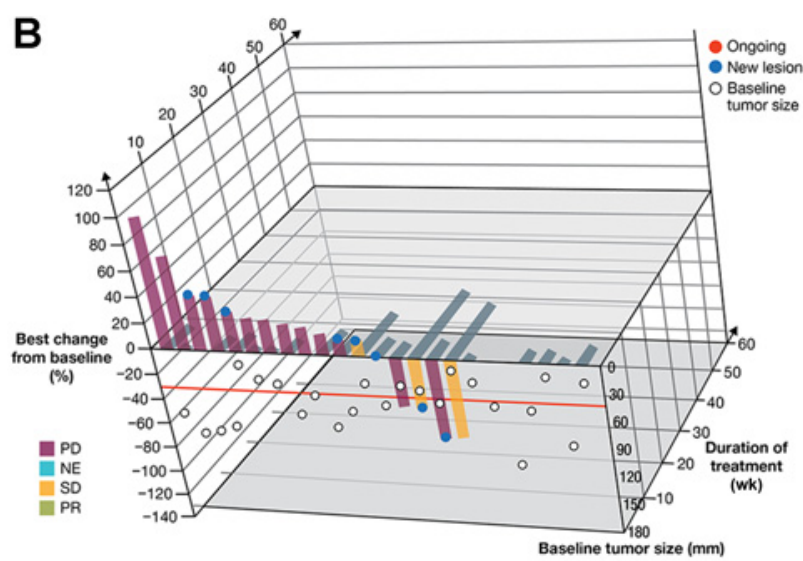

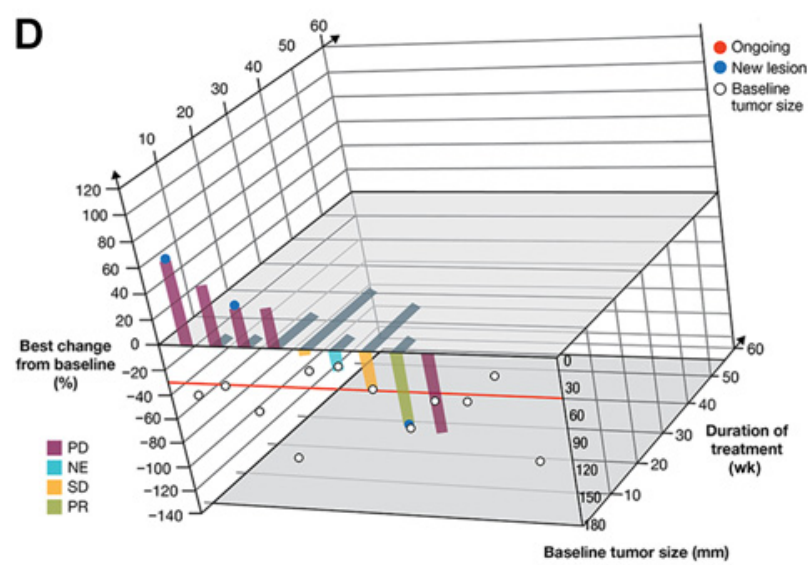

Figure 1.

Three-dimensional waterfall and swim plots of $\operatorname{arm} A(\mathbf{A}), \operatorname{arm} B(\mathbf{B}), \operatorname{arm} C(\mathbf{C}), \operatorname{arm} D(\mathbf{D})$, and $\operatorname{arm} E(\mathbf{E})$. The $x$-axis depicts individual patients; the $y$-axis depicts best change from baseline (\%). CR, PD, SD, PR, and SD were all per RECIST 1.1. Blue dot represents new lesion. The $z$-axis represents duration of treatment (weeks); red dot, ongoing therapy. White dot, baseline tumor size $(\mathrm{mm})$; NE, not evaluable.

In arm $\mathrm{D}$, in which 25 third-line patients were enrolled and received combination durvalumab and tremelimumab, the observed ORR was $4.0 \%$ ( 1 of 25 patients; $95 \%$ CI, $0.1 \%-20.4 \%$ ) and median OS was 9.2 months ( $95 \%$ CI, 5.4-12.6 months). The PFS rate at 6 months was $15.0 \%$ (95\% CI, $4.0 \%-32.6 \%)$. The OS rates at 12 and 24 months were $38.8 \%$ (95\% CI, $19.2 \%-58.1 \%)$ and $9.7 \%$ (95\% CI, $1.7 \%-26.5 \%$ ), respectively.
In arm E, 176 patients had tumor samples submitted for prescreening; 27 were ultimately screened. Nineteen patients were enrolled, with a median follow-up duration of 5.8 months. Among the samples prescreened for potential enrollment, the IFN $\gamma$ gene signature assay had a $70 \%$ success rate. The primary reason for failure was insufficient tissue resulting in insufficient nucleic acid available for RNA sequencing. The distribution of patients observed in the cohort was as 
Figure 2.

Overall survival.

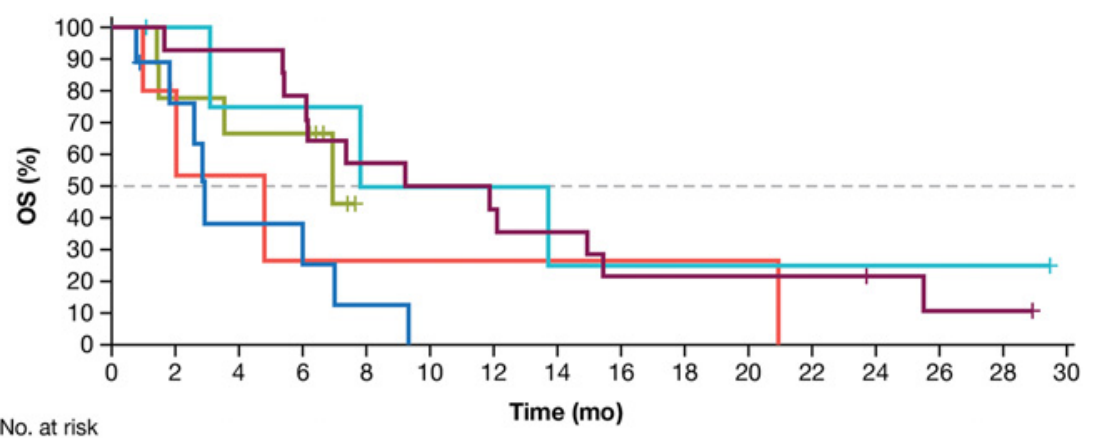

No. at risk

$\begin{array}{cccccccccccccccc}\text { Arm A } & 14 & 13 & 13 & 11 & 8 & 7 & 6 & 5 & 3 & 3 & 3 & 3 & 2 & 1 & 1 \\ \text { Arm B } & 9 & 6 & 3 & 3 & 1 & 0 & 0 & 0 & 0 & 0 & 0 & 0 & 0 & 0 & 0 \\ \text { Arm C } & 6 & 4 & 3 & 3 & 2 & 2 & 2 & 1 & 1 & 1 & 1 & 1 & 1 & 1 & 1 \\ \text { Arm D } & 5 & 3 & 2 & 1 & 1 & 1 & 1 & 1 & 1 & 1 & 1 & 0 & 0 & 0 & 0 \\ \text { Arm E } & 9 & 7 & 6 & 6 & 0 & 0 & 0 & 0 & 0 & 0 & 0 & 0 & 0 & 0 & 0\end{array}$

expected, with $37.5 \%$ of prescreened patients having an elevated signature and $62.5 \%$ not having elevated expression. There was one CR (5\%), two confirmed PRs (10.5\%), and one patient (5\%) with SD; the ORR was $15.8 \%$ (3 of 19 patients; $95 \%$ CI, 3.4\%-39.6\%). Duration of response was 13.3 weeks (range, 8.1-16.3 weeks). Median PFS was 1.8 months (95\% CI, 1.6-1.9 months), PFS rate at 6 months was $0 \%$ (95\% CI, NA), and median OS was 7.0 months (95\% CI, $2.4-$ 7.5 months). We correlated the degree of IFN $\gamma$ gene signature intensity with best overall response (Supplementary Fig. S3). Interestingly, in patients achieving CR or PR $(n=3)$, there was no difference in IFN $\gamma$ intensity ( -2.84 vs. $-2.83 ; P=0.99)$ compared with those who had progressive disease (PD; $n=14)$.

\section{Prognostic scores}

RMH and GRiM prognostic scores were retrospectively evaluated for all treatment arms. The proportion of those with low RMH scores $(0-1$ vs. $>1)$ was $77.8 \%, 37.5 \%, 75.0 \%, 80.0 \%$, and $63.2 \%$ for arms A through E, respectively, indicating that more patients enrolled in arms B and E were likely to have a poorer prognosis than patients in the other treatment arms. A lower percentage of low
GRiM scores ( $0-1$ vs. $>1)$ was observed in arms $\mathrm{B}$ and $\mathrm{E}$ than in other treatment arms $(92.6 \%, 70.8 \%, 91.7 \%, 84.0 \%$, and $68.4 \%$, respectively), which may explain in part the decreased OS rates observed in these patients.

\section{Biomarker evaluations}

MSI status was obtained for patients in all study cohorts (A-E), characterized using whole-exome sequencing (MSIsensor; ref. 25). A cutoff score of $\geq 10$ was used to define MSI-high (MSI-H; ref. 26). One patient in arm C was MSI-H (score 13) and was a nonresponder. All other patients $(n=72)$ were microsatellite stable (Fig. 3). PD-L1 status was available for $80.4 \%$ of patients (86/107). Clinical activity regarding PFS and OS was not affected by PD-L1 status (Supplementary Table S2). Furthermore, tumor mutation burden (TMB) was calculated using whole-exome sequencing. Median TMB in arms A-D was 2.13 mutations per megabase $(\mathrm{Mb})$. There was no correlation with PFS or OS when comparing high versus low TMB based on median, upper tertile, and upper quartile cutoff points. In addition, no differences were observed on the basis of response status (Supplementary Fig. S4). In arm E, median TMB was 5.00 mutations per Mb (range, 2.02-7.59;

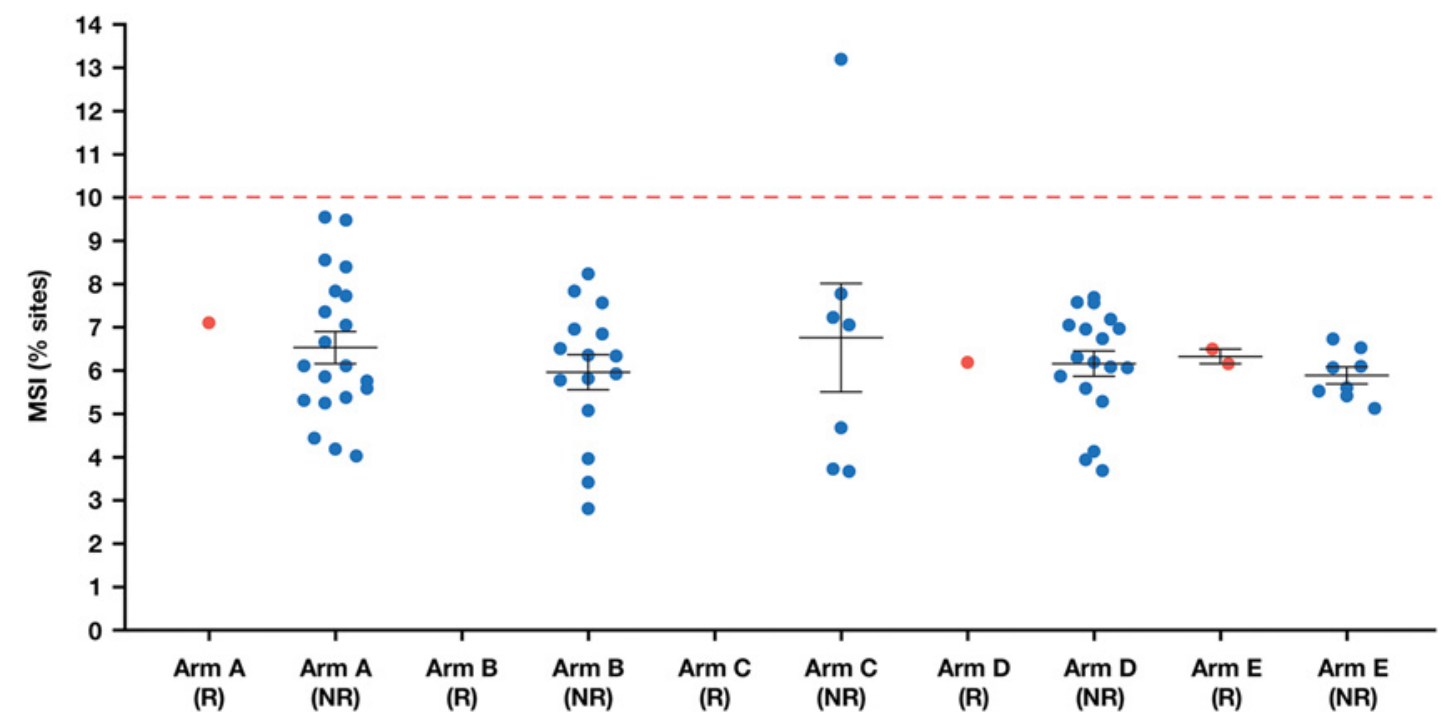

Figure 3.

MSI status based on responder status by arm. Data based on MSIsensor, which uses whole-exome sequencing for characterization of MSI status. Cut-off value $\geq 10$ designates MSI-H. Red dots, responders (R); blue dots, nonresponders (NR). 
Table 3. Treatment-related $A E s, \geq 10 \%$ any grade in any arm.

\begin{tabular}{|c|c|c|c|c|c|c|}
\hline \multirow[b]{2}{*}{ Adverse event } & \multicolumn{2}{|c|}{$\begin{array}{c}\text { Arms } A+D+E \\
2 L / 3 L D+T \\
(n=71)\end{array}$} & \multicolumn{2}{|c|}{$\begin{array}{c}\text { Arm B } \\
\text { 2L D } \\
(n=24)\end{array}$} & \multicolumn{2}{|c|}{$\begin{array}{c}\text { Arm C } \\
2 \mathrm{~L} T \\
(n=12)\end{array}$} \\
\hline & Any Grade & Grade $3 / 4$ & Any Grade & Grade $3 / 4$ & Any Grade & Grade $3 / 4$ \\
\hline Diarrhea & $12(16.9)$ & $2(2.8)$ & $1(4.2)$ & 0 & $3(25.0)$ & $1(8.3)$ \\
\hline Aspartate aminotransferase increased & $2(2.8)$ & 0 & 0 & 0 & $3(25.0)$ & $1(8.3)$ \\
\hline Dyspnea & $4(5.6)$ & 0 & 0 & 0 & $3(25.0)$ & 0 \\
\hline Fatigue & $14(19.7)$ & $2(2.8)$ & $2(8.3)$ & 0 & $2(16.7)$ & $1(8.3)$ \\
\hline Decreased appetite & $11(15.5)$ & $1(1.4)$ & $3(12.5)$ & 0 & $1(8.3)$ & 0 \\
\hline Colitis & $4(5.6)$ & $3(4.2)$ & 0 & 0 & $2(16.7)$ & $2(16.7)$ \\
\hline Pruritus & $8(11.3)$ & 0 & 0 & 0 & $2(16.7)$ & 0 \\
\hline Rash $^{a}$ & $9(12.7)$ & $1(1.4)$ & 0 & 0 & $2(16.7)$ & 0 \\
\hline Alanine aminotransferase increased & $1(1.4)$ & $1(1.4)$ & 0 & 0 & $2(16.7)$ & 0 \\
\hline Lipase increased & $2(2.8)$ & $2(2.8)$ & 0 & 0 & $2(16.7)$ & $1(8.3)$ \\
\hline Dermatitis acneiform & $1(1.4)$ & 0 & 0 & 0 & $2(16.7)$ & $1(8.3)$ \\
\hline Nausea & $6(8.5)$ & $1(1.4)$ & $1(4.2)$ & 0 & $1(8.3)$ & 0 \\
\hline Dry skin & $2(2.8)$ & 0 & 0 & 0 & $2(16.7)$ & 0 \\
\hline Amylase increased & $2(2.8)$ & $2(2.8)$ & 0 & 0 & $2(16.7)$ & 0 \\
\hline Vomiting & $4(5.6)$ & 0 & 0 & 0 & $1(8.3)$ & 0 \\
\hline Weight decreased & $4(5.6)$ & 0 & 0 & 0 & 0 & 0 \\
\hline Hyperglycemia & $3(4.2)$ & $2(2.8)$ & 0 & 0 & 0 & 0 \\
\hline Blood thyroid-stimulating hormone increased & $3(5.8)$ & 0 & 0 & 0 & 0 & 0 \\
\hline Hypothyroidism & $3(5.8)$ & 0 & 0 & 0 & 0 & 0 \\
\hline
\end{tabular}

apooled data for rash and rash maculopapular.

$n=11$ ). Similar to other arms, there was no correlation of TMB with response status.

ctDNA samples were obtained from 72 patients at baseline and 31 patients at both baseline and 9 weeks on treatment. Patients with PR showed a decrease $(\Delta,-10.05 \% ; P=0.26)$ in VAF posttreatment, compared with a significant increase in mean VAF in patients with $\mathrm{PD}(\Delta,+8.14 \% ; P=0.03$; Supplementary Fig. S5). This correlation was also observed in total mutation count in patients with $\mathrm{PR}(\Delta,-6.7$; $P=0.17)$ compared with patients with $\operatorname{PD}(\Delta,+2.2 ; P=0.06)$. Patients with a decrease in VAF at week 9 had longer median PFS [5.4 months; 95\% CI, 3.7-not reached (NR; ref. 27) and OS (median, 13.8 months; 95\% CI, 7.0-NR) than those with an increase in VAF [median PFS, 1.9 months (95\% CI, $1.8-\mathrm{NR})$; HR, 0.33 ; 95\% CI, 0.09-1.21] and median OS, 8.2 months (95\% CI, 6.2-NR); HR, 0.07; 95\% CI, 0.010.66]. In addition, by using ctDNA, KRAS mutation (nonsynonymous single variant nucleotide) was identified in 2 of 113 patients $(1.8 \%)$, both of whom had PD.

\section{Safety and tolerability}

In the phase $\mathrm{Ib}$ portion, one of six patients had a dose-limiting toxicity of grade 3 hepatic function abnormality (transaminitis), which was initially attributed as possibly related to the investigational drug; further evaluation revealed that the patient had disease progression of liver metastases. The dose of $20 \mathrm{mg} / \mathrm{kg}$ durvalumab and $1 \mathrm{mg} / \mathrm{kg}$ tremelimumab was confirmed safe and tolerable for the phase II portion.

In the phase II part of this study, the proportion of patients reporting one or more TRAE was $33.3 \%$ in the durvalumab monotherapy arm and $66.7 \%$ in the tremelimumab monotherapy arm. The incidence of AEs varied between the combination treatment arms (A, 70.4\%; D, $56.0 \%$; E, $42.1 \%$ ). The most frequently observed TRAEs ( $\geq 10 \%$ any grade in any treatment arm) included fatigue, diarrhea, decreased appetite, and pruritus (Table 3). TRAEs leading to treatment discontinuation occurred in $14.8 \%(4 / 27), 20.0 \%(5 / 25)$, and $5.3 \%(1 / 19)$ in the three combination therapy arms (arms A, D, and E), compared with $33.3 \%$ of patients $(4 / 12)$ in the tremelimumab monotherapy arm and $4.2 \%$ of patients $(1 / 24)$ in the durvalumab monotherapy arm. No TRAEs leading to death were observed.

\section{Discussion}

This completed phase Ib/II study is the first to evaluate a PD-L1 and CTLA-4 combination strategy in metastatic gastric cancer/GEJ adenocarcinoma and shows that the combination of durvalumab and tremelimumab demonstrated numerically higher rates of confirmed response than durvalumab monotherapy. Twelve-month OS rates in the combined therapy arms were comparable with the combination of nivolumab with ipilimumab in the CheckMate 032 study (37\% vs. $35 \%$; ref. 6). As anticipated, the incidence of TRAEs was higher in the combination arm and the tremelimumab monotherapy arm than in the durvalumab monotherapy arm.

In this study, we prospectively screened patients with a novel RNA-based IFN $\gamma$ gene expression signature comprising IFN $\gamma, C D 274$, $L A G 3$, and CXCL9. We found this approach to be feasible for patient selection with predictable assay performance. Although the ORRs with durvalumab and tremelimumab were higher in the IFN $\gamma$ signaturehigh group than those in unselected populations, survival outcomes remained similar. One important distinction between the assessment of the IFN $\gamma$ gene signature in this study and that used in previous studies (15) was the use of archival tissue rather than fresh tissue. We postulate that IFN $\gamma$ is a dynamic marker and scores can vary based on multiple factors, including timing of tissue acquisition, number of previous lines of therapy, and location of tissue acquisition (primary tumor vs. metastatic site). In this study, archival tissue was used due to availability and for pragmatic reasons to prevent delay in initiation of therapy in this fragile patient population. Given the added complexity of patient preselection and lack of substantial improvement in clinical outcomes, these results do not support further implementation 
of the IFN $\gamma$ gene signature for patient selection, as designed, in this population.

The use of PD-L1 expression as a biomarker for patient selection in advanced gastric cancer/GEJ cancer continues to evolve. In studies of pembrolizumab, PD-L1 status was assessed using the combined positive score (CPS), which counts PD-L1 staining in both tumor and peritumoral mononuclear cells (5). With the CPS, PD-L1negative (CPS 0) tumors are associated with worse outcomes than PD-L1-positive tumors (CPS $\geq 1$ ). These findings are consistent with a recent meta-analysis, suggesting that $\mathrm{PD}-\mathrm{L} 1$ expression levels are associated with OS (28). However, in the current study, no clear difference in clinical activity based on PD-L1 status was observed. This may be explained by our use of PD-L1 staining in tumor cells only, as well as differences in patient cohorts and immunohistochemistry antibodies.

To validate additional biomarkers, we evaluated pretreatment and on-treatment levels of ctDNA in a subset of patients in this study. Although our sample size was small, ctDNA VAFs were consistently reduced after 9 weeks of therapy in responders but not in nonresponders. A decrease in VAF following 9 weeks of treatment correlated with longer PFS and OS, suggesting utility as an early indicator of clinical benefit (Supplementary Fig. S5). These findings have also been observed independently in other cancers and suggest that ctDNA may be a promising biomarker of response to ICIs in gastric cancer. Importantly, the utilization of ctDNA VAF changes should be evaluated in future studies as a method of determining response to treatment rather than relying solely on radiographic changes as measured by the RECIST criteria. This is particularly because clinical responses may be delayed and can infrequently be preceded by apparent progression (so-called pseudoprogression).

One possibility for the differences in efficacy observed in arm B (durvalumab monotherapy) and arm E (IFN $\gamma$ signature-high, durvalumab plus tremelimumab) compared with the other study arms may be a higher rate of enrolled patients with poor prognostic factors, as measured by RMH and GRIm scores. In a separate phase I trial, durvalumab monotherapy demonstrated a higher rate of OS in patients with eligibility characteristics similar to those in this study (17). The possibility that patients with more adverse features were enrolled in this study is supported by the observation that only 1 of 72 evaluated patients (1.4\%) had an MSI-high tumor; this is lower than the $4 \%$ incidence normally noted in patients with metastatic disease and the $12 \%$ of patients (7/59) treated with nivolumab alone in CheckMate 032 (6).

A limitation of this study is the relatively small sample size in each cohort, which affected the statistical power and precluded the ability to distinguish between treatment arms. Per protocol, enrollment was stopped following interim analyses of efficacy. In addition, although pembrolizumab was active in patients who were MSI-H and those with EBV-associated gastric cancer/GEJ cancer (29), we are unable to conclude how the therapies in our study would perform in these specific populations. As mentioned above, there was a very low proportion of MSI-H patients. EBV status was not available, as testing viral status was not a standard practice at the time of this trial. Furthermore, a lack of available tissue precluded EBV testing retrospectively.

In summary, this is the second trial to investigate the efficacy of combination checkpoint inhibitors in gastric cancer/GEJ cancer and the first to assess a PD-L1 inhibitor with a CTLA- 4 inhibitor. Although responses rates with durvalumab or tremelimumab monotherapy or in combination were low, the combination approach resulted in a 12month OS rate of approximately $37 \%$. These results highlight that a better understanding of the underlying immune microenvironment is paramount to prospectively identify which patients should receive combined checkpoint inhibitors. Biomarker strategies beyond PD-L1 expression, MSI status, and the use of IFN gene expression are ongoing.

\section{Disclosure of Potential Conflicts of Interest}

R.J. Kelly is an advisory board member/paid consultant for AstraZeneca, BristolMyers Squibb, Merck, Eli Lilly, Astellas, and Novartis. Y. Bang is an employee/paid consultant for AstraZeneca, Novartis, Genentech/Roche, MSD, Merck Serano, Bayer, Bristol-Myers Squibb, Eli Lilly, Taiho, Daiichi-Sankyo, Astellas, BeiGene, GreenCross, Samyang Biopharm, Hanmi, Genexine, and GlaxoSmithKline, and reports receiving commercial research grants from AstraZeneca, Novartis, Genentech/Roche, MSD, Merck Serano, Bayer, Bristol-Myers Squibb, GlaxoSmithKline, Pfizer, Eli Lilly, Boeringer-Ingelheiim, Macrogenics, Boston Biomedical, FivePrime, Curis, Taiho, Takeda, Ono, Daiichi-Sankyo, Astellas, BeiGene, GreenCross, CKD Biopharma, and Genexine. D.V.T. Catenacci is an employee/paid consultant for Bristol-Myers Squibb, Merck, Taiho, Gritstone, Lilly, Genentech/Roche, Seattle Genetics, and Daichii Sankyo, and reports receiving speakers bureau honoraria from Foundation Medicine, Merck, Lilly, Guardant Health, Tempus, and Genentech/Roche. Zev A. Wainberg is an employee/paid consultant for AstraZeneca, Bayer, Merck, Lilly, and Bristol-Myers Squibb. J.C. Bendell is an employee/paid consultant for Gilead, Genetech/Roche, Bristol-Myers Squibb, Five Prime, Lilly, Merck, MedIumme, Celgene, Taiho, Macrogenics, GlaxoSmithKline, Novartis, OncoMed, LEAP, TG Thearpeutics, AstraZenca, BI, Daiichi Sankyo, Bayer, Incyte, Apexigen, Array, Sanofi, ARMO, Ipsen, Merrimack, Oncogenex, FORMA, Arch Oncology, Prelude Therapeutics, Phoenix Bio, Cyteir, Molecular Partners, Innate, Torque, Tizona, Janssen, Tolero, Translational Drug Development, Amgen, Settle Genetics, Moderna therapeutics, Tanabe Research Laboratories, Beigene, Continuum Clinical, and Agois, reports receiving commercial research grants from Gilead, Genentech/Roche, Bristol-Myers Squibb, Five Prime, Lilly, Merck, MedImmune, Celgene, EMD Serono, Taiho, Marcogenics, GlaxoSmithKline, Novartis, OncMed, LEAP, TG Therapeutics, AstraZenca, BI, Daiichi Sankyo, Bayer, Incyte, Apexigen, Koltan, SynDevRex, Forty Seven, AbbVie, Array, Onyx, Sanofi, Takeda, Eisai, Celldex, Agios, Cytomx, Nektar, ARMO, Boston Biomedical, Ispen, Merrimack, Tarveda, Tyrogenex, Oncogenex, Marshall Edwards, Pieries, Mersana, Calithera, Blueprint, Evelo, FORMA, Merus, Jacobio, Effector, Novocare, Arrys, Tracon, Sierra, Innate, Arch Oncology, Prelude Oncology, Unum Therapeutics, Vyriad, Harpoon, ADC, Amgen, Pfizer, Millennium, ImClone, Acerta Pharma, Rgenix, Bellicum, Gossamer Bio, and Arcus Bio, and other remuneration from Gilead, Genetech/Roche, Bristol-Myers Squibb, Lilly, Merck, Medimmune, Celgene, Taiho, Novartis, OncoMed, BI, ARMO, Ipsen, Oncogenex, and FORMA C.S. Denlinger is an employee/paid consultant for Merck, Bristol-Myers Squibb, Eli Lilly, Astellas, Exelixis, QED Therapeutics, Carevive, Bayer, and Beigene. R. Leidner reports receiving commercial research grants from Bristol-Myers Squibb. H. Lenz is an advisory board member/unpaid consultant for Bristol-Myers Squibb, Merck KG, and Bayer. M.C. Rebelatto is an employee/paid consultant for and holds ownership interest (including patents) in AstraZeneca. P. Brohawn is an employee/paid consultant for AstraZeneca and Immunocore Ltd. P. He is an employee/paid consultant for AstraZeneca. J. McDevitt is an employee/paid consultant for Medimmune. J.M. Englert is an employee/paid consultant for Medimmune/AstraZeneca. G.Y. Ku is an employee/paid consultant for Merck, Pieris, Bristol-Myers Squibb, and Eli Lilly, reports receiving commercial research grants from AstraZeneca and Arog, other commercial research support from Bristol-Myers Squibb, Pieris, and Zymeworks, and speakers bureau honoraria from Dava Oncology. No potential conflicts of interest were disclosed by the other authors.

\section{Authors' Contributions}

Conception and design: R.J. Kelly, Y.-J. Bang, M. Blum-Murphy, Y. Chao, M.C. Rebelatto, J. McDevitt, J. Englert

Development of methodology: R.J. Kelly, Y.-J. Bang, M.C. Rebelatto, P.Z. Brohawn, J. McDevitt, J. Englert

Acquisition of data (provided animals, acquired and managed patients, provided facilities, etc.): R.J. Kelly, J. Lee, Y.-J. Bang, K. Almhanna, M. Blum-Murphy, D. Catenacci, H.C. Chung, Z.A. Wainberg, M.K. Gibson, K.-W. Lee, J. Bendell, C.S. Denlinger, C.E. Chee, R. Leidner, H.-J. Lenz, P.Z. Brohawn, S. Sheth, G.Y. Ku Analysis and interpretation of data (e.g., statistical analysis, biostatistics, computational analysis): R.J. Kelly, Y.-J. Bang, K. Almhanna, D. Catenacci, H.C. Chung, Z.A. Wainberg, M.K. Gibson, C.S. Denlinger, H.-J. Lenz, Y. Chao, M.C. Rebelatto, P.Z. Brohawn, P. He, J. McDevitt, S. Sheth, J. Englert, G.Y. Ku Writing, review, and/or revision of the manuscript: R.J. Kelly, Y.-J. Bang, K. Almhanna, M. Blum-Murphy, D. Catenacci, H.C. Chung, Z.A. Wainberg, 


\section{Kelly et al.}

M.K. Gibson, K.-W. Lee, J. Bendell, C.S. Denlinger, C.E. Chee, T. Omori, R. Leidner, H.-J. Lenz, M.C. Rebelatto, P.Z. Brohawn, J. McDevitt, S. Sheth, J. Englert, G.Y. Ku Administrative, technical, or material support (i.e., reporting or organizing data, constructing databases): H.-J. Lenz, Y. Chao, S. Sheth, J. Englert

Study supervision: R.J. Kelly, C.S. Denlinger, J. McDevitt, J. Englert

\section{Acknowledgments}

The authors thank the patients, their families and caregivers, and the site investigators and staff for their participation in this study, and Christopher Morehouse of AstraZeneca for performing biomarker analyses. This study was funded by
AstraZeneca. Medical writing support was provided by Ailsa Bennett, PhD, of SciMentum, Inc. (Nucleus Global), London, UK, and was funded by AstraZeneca.

The costs of publication of this article were defrayed in part by the payment of page charges. This article must therefore be hereby marked advertisement in accordance with 18 U.S.C. Section 1734 solely to indicate this fact.

Received July 26, 2019; revised October 3, 2019; accepted October 29, 2019; published first November 1, 2019.

\section{References}

1. Digklia A, Wagner AD. Advanced gastric cancer: current treatment landscape and future perspectives. World J Gastroenterol 2016;22:2403-14.

2. Salati M, Di Emidio K, Tarantino V, Cascinu S. Second-line treatments: moving towards an opportunity to improve survival in advanced gastric cancer? ESMO Open 2017;2:e000206

3. Ghosn M, Tabchi S, Kourie HR, Tehfe M. Metastatic gastric cancer treatment: second line and beyond. World J Gastroenterol 2016;22:3069-77.

4. Bang YJ, Cho JY, Kim YH, Kim JW, Di Bartolomeo M, Ajani JA, et al. Efficacy of sequential ipilimumab monotherapy versus best supportive care for unresectable locally advanced/metastatic gastric or gastroesophageal junction cancer. Clin Cancer Res 2017;23:5671-8.

5. Fuchs CS, Doi T, Jang RW, Muro K, Satoh T, Machado M, et al. Safety and efficacy of pembrolizumab monotherapy in patients with previously treated advanced gastric and gastroesophageal junction cancer: phase 2 clinical KEYNOTE-059 trial. JAMA Oncol 2018;4:e180013.

6. Janjigian YY, Bendell J, Calvo E, Kim JW, Ascierto PA, Sharma P, et al CheckMate-032 study: efficacy and safety of nivolumab and nivolumab plus ipilimumab in patients with metastatic esophagogastric cancer. J Clin Oncol 2018;36:2836-44.

7. Kang YK, Boku N, Satoh T, Ryu MH, Chao Y, Kato K, et al. Nivolumab in patients with advanced gastric or gastro-oesophageal junction cancer refractory to, or intolerant of, at least two previous chemotherapy regimens (ONO-4538-12, ATTRACTION-2): a randomised, double-blind, placebo-controlled, phase 3 trial. Lancet 2017;390:2461-71.

8. Kelly RJ. Immunotherapy for esophageal and gastric cancer. Am Soc Clin Oncol Educ Book 2017;37:292-300.

9. Shitara K, Ozguroglu M, Bang YJ, Di Bartolomeo M, Mandala M, Ryu MH, et al. Pembrolizumab versus paclitaxel for previously treated, advanced gastric or gastro-oesophageal junction cancer (KEYNOTE-061): a randomised, openlabel, controlled, phase 3 trial. Lancet 2018;392:123-33.

10. Thompson ED, Zahurak M, Murphy A, Cornish T, Cuka N, Abdelfatah E, et al. Patterns of PD-L1 expression and CD8 T cell infiltration in gastric adenocarcinomas and associated immune stroma. Gut 2017;66:794-801.

11. Derks S, Liao X, Chiaravalli AM, Xu X, Camargo MC, Solcia E, et al. Abundant PD-L1 expression in Epstein-Barr virus-infected gastric cancers. Oncotarget 2016;7:32925-32

12. Zhu L, Li Z, Wang Y, Zhang C, Liu Y, Qu X. Microsatellite instability and survival in gastric cancer: a systematic review and meta-analysis. Mol Clin Oncol 2015;3: 699-705.

13. The Cancer Genome Atlas Research Network. Comprehensive molecular characterization of gastric adenocarcinoma. Nature 2014;513:202-9.

14. Ayers M, Lunceford J, Nebozhyn M, Murphy E, Loboda A, Kaufman DR, et al. IFN-gamma-related mRNA profile predicts clinical response to $\mathrm{PD}-1$ blockade. J Clin Invest 2017;127:2930-40.

15. Higgs BW, Morehouse CA, Streicher K, Brohawn PZ, Pilataxi F, Gupta A, et al. Interferon gamma messenger RNA signature in tumor biopsies predicts out-

comes in patients with non-small cell lung carcinoma or urothelial cancer treated with durvalumab. Clin Cancer Res 2018;24:3857-66.

16. Antonia S, Goldberg SB, Balmanoukian A, Chaft JE, Sanborn RE, Gupta A, et al. Safety and antitumour activity of durvalumab plus tremelimumab in non-small cell lung cancer: a multicentre, phase lb study. Lancet Oncol 2016;17:299-308.

17. Hollebecque A, Wainberg ZA, Ajani JA, Marshall J, Cunningham D, Ou S-H, et al. Safety and clinical activity of durvalumab monotherapy in patients with gastroesophageal cancers. J Clin Oncol 36:15s, 2018 (suppl; abstr 4032).

18. Powles T, O'Donnell PH, Massard C, Arkenau HT, Friedlander TW, Hoimes CI, et al. Efficacy and safety of durvalumab in locally advanced or metastatic urothelial carcinoma: updated results from a phase $1 / 2$ open-label study. JAMA Oncol 2017;3:e172411.

19. Stewart R, Morrow M, Hammond SA, Mulgrew K, Marcus D, Poon E, et al Identification and characterization of MEDI4736, an antagonistic anti-PD-L1 monoclonal antibody. Cancer Immunol Res 2015;3:1052-62.

20. Ralph C, Elkord E, Burt DJ, O'Dwyer JF, Austin EB, Stern PL, et al. Modulation of lymphocyte regulation for cancer therapy: a phase II trial of tremelimumab in advanced gastric and esophageal adenocarcinoma. Clin Cancer Res 2010;16: 1662-72.

21. National Cancer Institute. NCI Common Terminology Criteria for Adverse Events (CTCAE) v4.0. Bethesda, MD; National Institutes of Health 2009.

22. Arkenau HT, Barriuso J, Olmos D, Ang JE, de Bono J, Judson I, et al. Prospective validation of a prognostic score to improve patient selection for oncology phase I trials. J Clin Oncol 2009;27:2692-6.

23. Bigot F, Castanon E, Baldini C, Hollebecque A, Carmona A, Postel-Vinay S, et al Prospective validation of a prognostic score for patients in immunotherapy phase I trials: the Gustave Roussy Immune Score (GRIm-Score). Eur J Cancer 2017;84:212-8.

24. Wilke H, Muro K, Van Cutsem E, Oh SC, Bodoky G, Shimada Y, et al. Ramucirumab plus paclitaxel versus placebo plus paclitaxel in patients with previously treated advanced gastric or gastro-oesophageal junction adenocarcinoma (RAINBOW): a double-blind, randomised phase 3 trial. Lancet Oncol 2014;15:1224-35.

25. Niu B, Ye K, Zhang Q, Lu C, Xie M, McLellan MD, et al. MSIsensor: microsatellite instability detection using paired tumor-normal sequence data. Bioinformatics 2014;30:1015-6.

26. Middha S, Zhang L, Nafa K, Jayakumaran G, Wong D, Kim HR, et al. Reliable pan-cancer microsatellite instability assessment by using targeted nextgeneration sequencing data. JCO Precis Oncol 2017;1:1-17.

27. Dunn GP, Koebel CM, Schreiber RD. Interferons, immunity and cancer immunoediting. Nat Rev Immunol 2006;6:836-48.

28. Gu L, Chen M, Guo D, Zhu H, Zhang W, Pan J, et al. PD-L1 and gastric cancer prognosis: a systematic review and meta-analysis. PLoS One 2017;12:e0182692.

29. Kim ST, Cristescu R, Bass AJ, Kim KM, Odegaard JI, Kim K, et al. Comprehensive molecular characterization of clinical responses to PD-1 inhibition in metastatic gastric cancer. Nat Med 2018;24:1449-58. 


\section{Clinical Cancer Research}

\section{Safety and Efficacy of Durvalumab and Tremelimumab Alone or in Combination in Patients with Advanced Gastric and Gastroesophageal Junction Adenocarcinoma}

Ronan J. Kelly, Jeeyun Lee, Yung-Jue Bang, et al.

Clin Cancer Res 2020;26:846-854. Published OnlineFirst November 1, 2019.

\section{Updated version Access the most recent version of this article at: doi:10.1158/1078-0432.CCR-19-2443}

Supplementary Access the most recent supplemental material at:

Material http://clincancerres.aacrjournals.org/content/suppl/2019/11/01/1078-0432.CCR-19-2443.DC1

Cited articles This article cites 27 articles, 6 of which you can access for free at: http://clincancerres. aacrjournals.org/content/26/4/846.full\#ref-list-1

E-mail alerts Sign up to receive free email-alerts related to this article or journal.

Reprints and To order reprints of this article or to subscribe to the journal, contact the AACR Publications Department at Subscriptions pubs@aacr.org.

Permissions To request permission to re-use all or part of this article, use this link http://clincancerres.aacrjournals.org/content/26/4/846.

Click on "Request Permissions" which will take you to the Copyright Clearance Center's (CCC) Rightslink site. 